Supporting information for:

\title{
Platinum Nanoparticle Modified Single-Walled Carbon Nanotube-Laden Paper Electrodes for Electrocatalytic Oxidation of Methanol
}

\author{
Janak Paudyal, ${ }^{1,4 \dagger}$ Po Wang, ${ }^{1,2 \dagger}$ Fuyi Zhou, ${ }^{2}$ Yingzhu Liu, ${ }^{1}$ Yong Cai ${ }^{1}$ and Yi Xiao ${ }^{1,3 *}$
}

${ }^{1}$ Department of Chemistry and Biochemistry, Florida International University, $11200 \mathrm{SW} 8$ th Street, Miami, FL, 33199. ${ }^{2}$ School of Chemistry and Chemical Engineering, Jiangsu Normal University, Xuzhou 221116, China; ${ }^{3}$ Department of Chemistry, North Carolina State University, 2620 Yarbrough Dr., Raleigh, NC, USA, 27695. ${ }^{4}$ Department of Chemistry and Biochemistry, University of Colorado - Colorado Springs, 1420 Austin Bluffs Pkwy, Colorado Springs, CO, 80918.

$\uparrow$ These authors contributed equally to this work.

*Corresponding author: yxiao34@ncsu.edu 


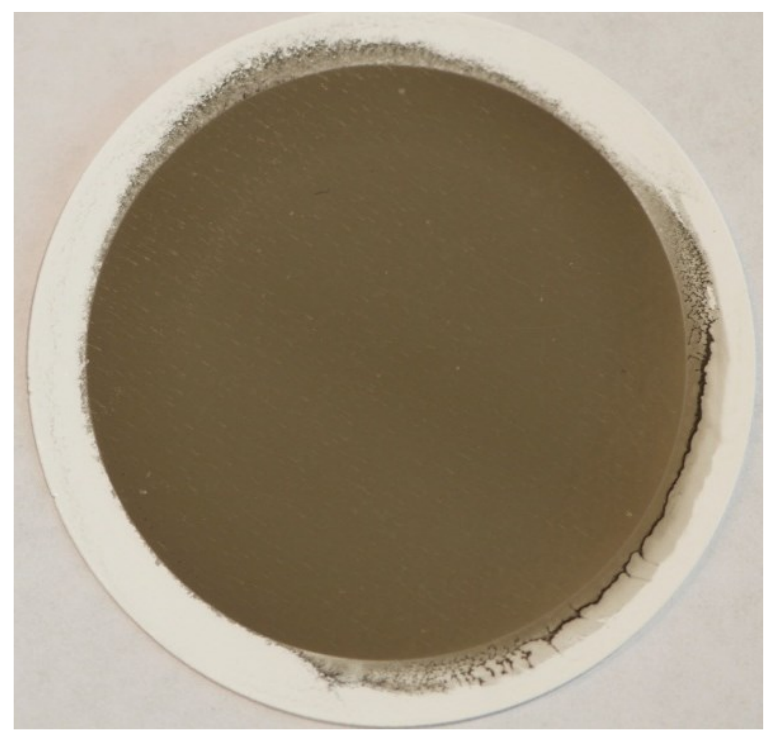

SWCNT thin film

Figure S1. Photograph of SWCNT thin film prepared via ambient vacuum filtration.
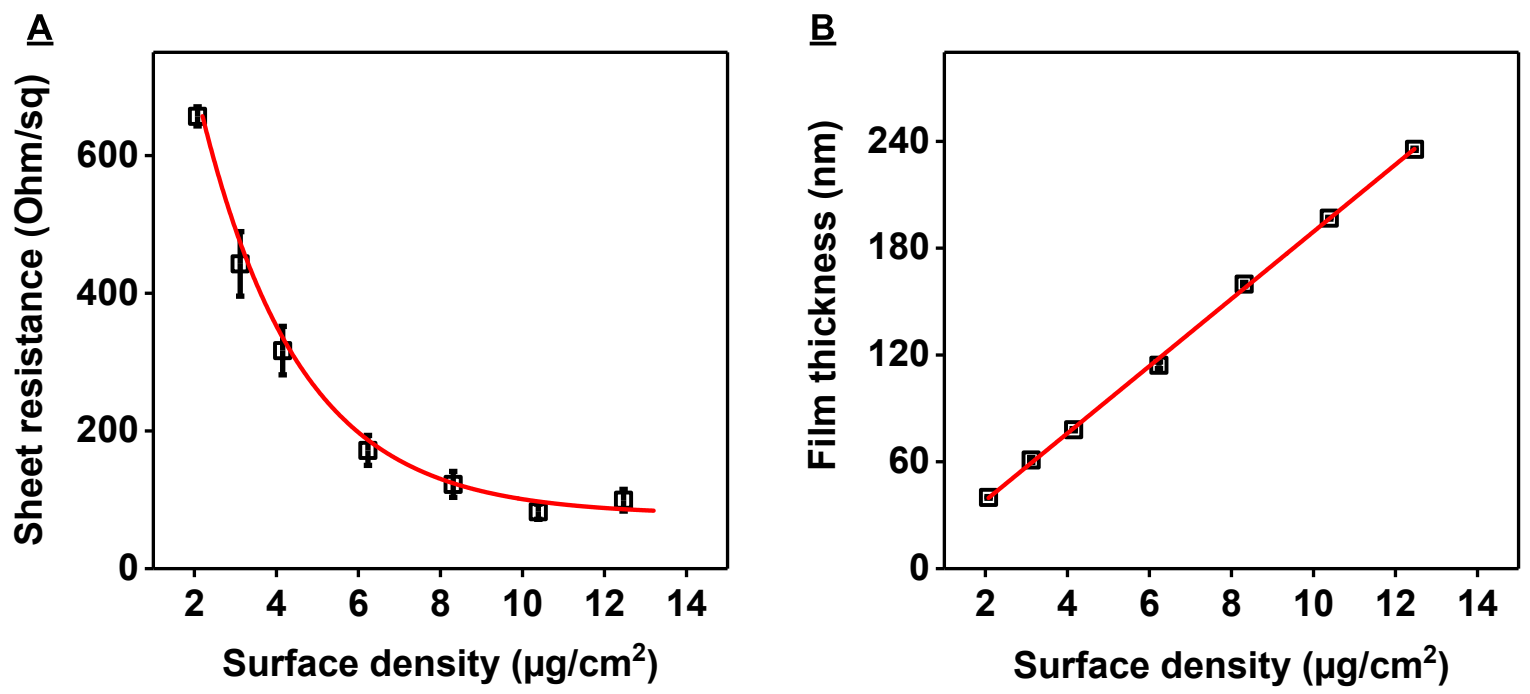

Figure S2. Characterization of paper-based SWCNT thin film prepared using vacuum filtration. Effect of surface density of SWCNTs on (A) sheet resistance or (B) film thickness. 
(A) $2.1 \mu \mathrm{g} / \mathrm{cm}^{2}$

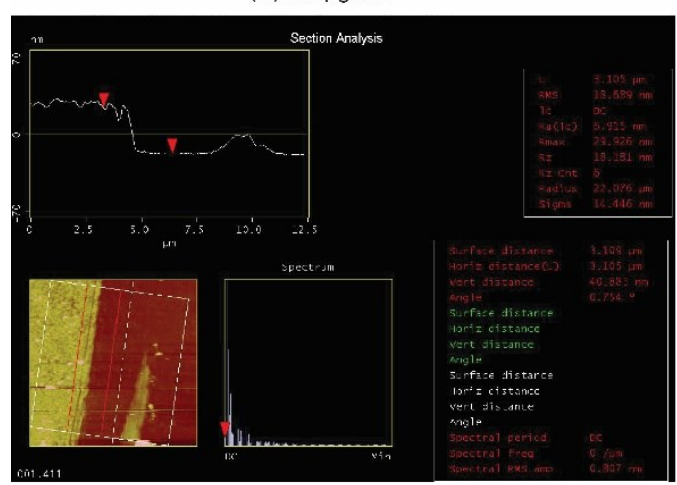

(C) $4.2 \mu \mathrm{g} / \mathrm{cm}^{2}$

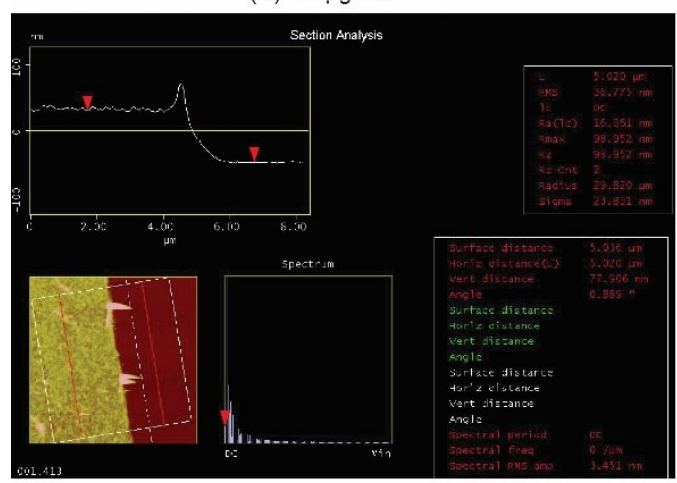

(E) $8.3 \mu \mathrm{g} / \mathrm{cm}^{2}$

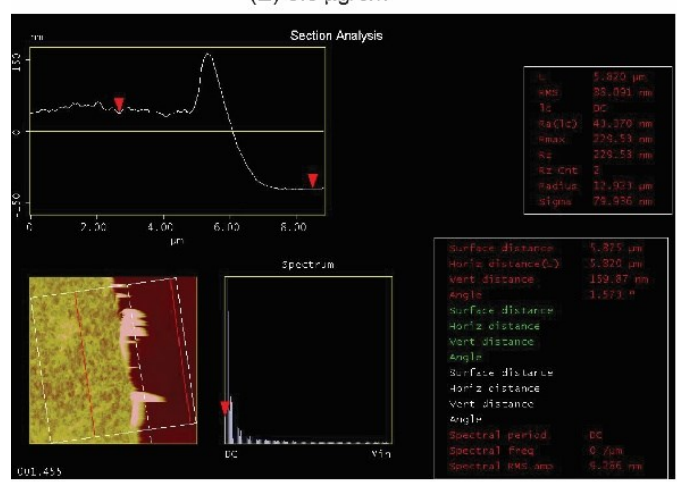

(B) $3.1 \mu \mathrm{g} / \mathrm{cm}^{2}$

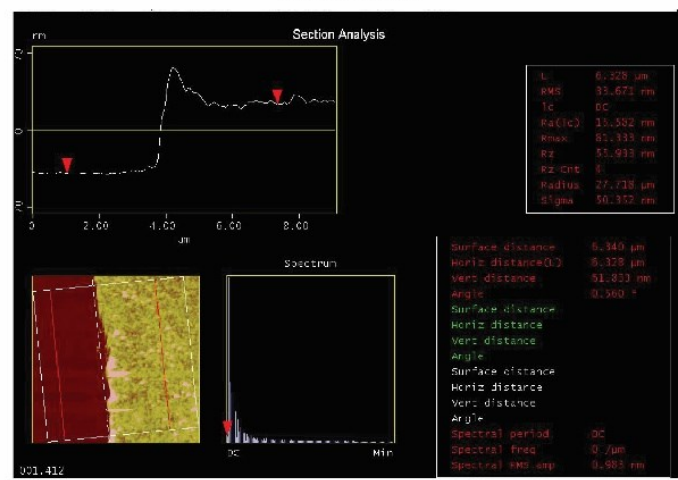

(D) $6.2 \mu \mathrm{g} / \mathrm{cm}^{2}$

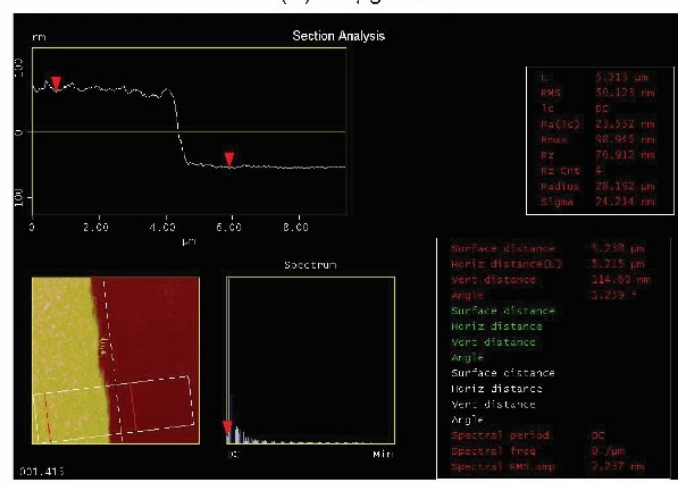

(F) $10.4 \mu \mathrm{g} / \mathrm{cm}^{2}$

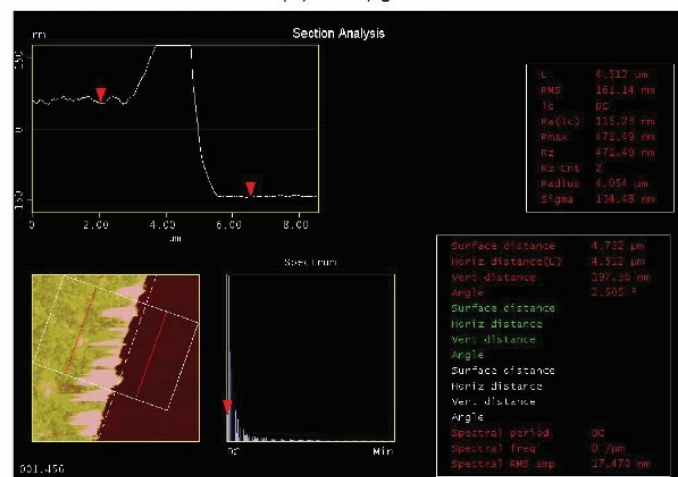

(G) $12.5 \mu \mathrm{g} / \mathrm{cm}^{2}$

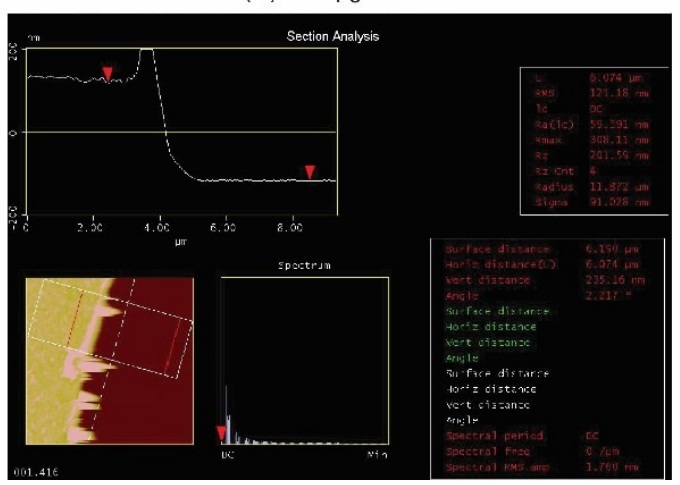

Figure S3. Measurement of thickness of SWCNT thin films using atomic force microscopy (AFM). Panels show AFM measurements for the films prepared with surface densities of SWCNTs at (A) 2.1, (B) 3.1, (C) 4.2, (D) 6.2, (E) 8.3, (F) 10.4, and (G) $12.5 \mu \mathrm{g} / \mathrm{cm}^{2}$. 


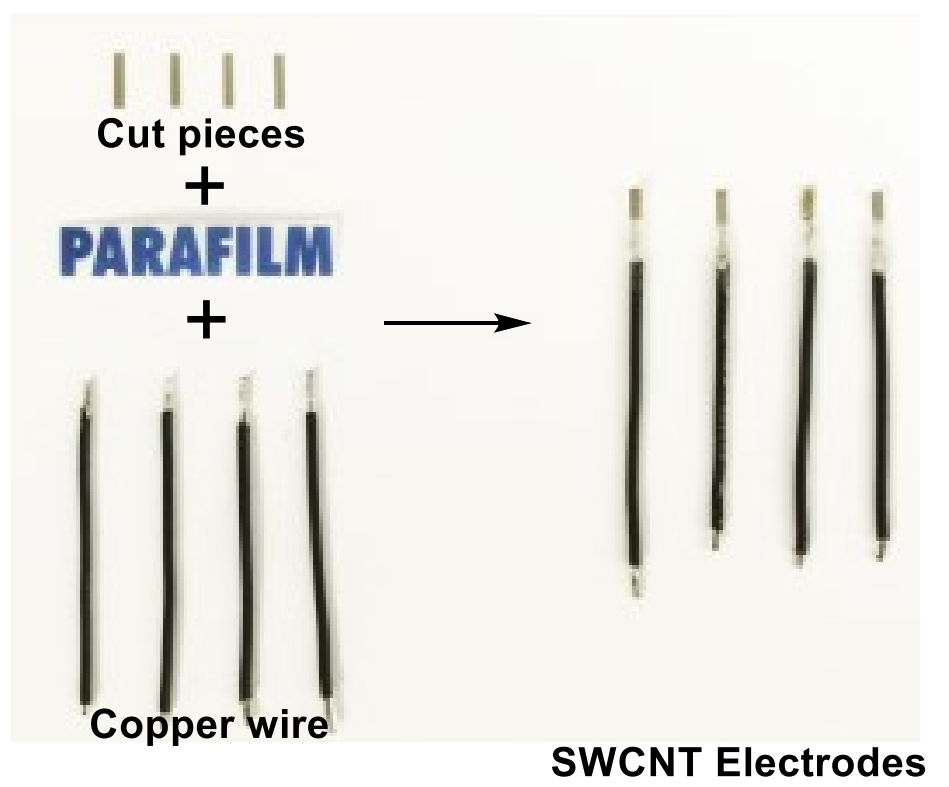

Figure S4. Schematic of fabrication of SWCNT paper electrodes from thin SWCNT films.

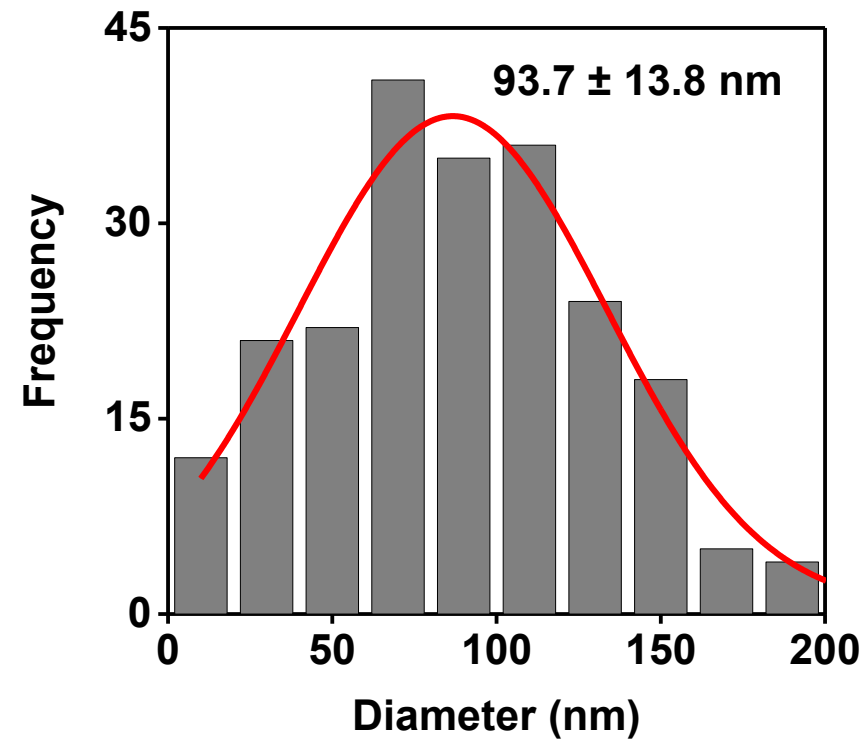

Figure S5. Size distribution of PtNCs from the image shown in Figure $2 \mathrm{C}$ as analyzed using ImageJ. 


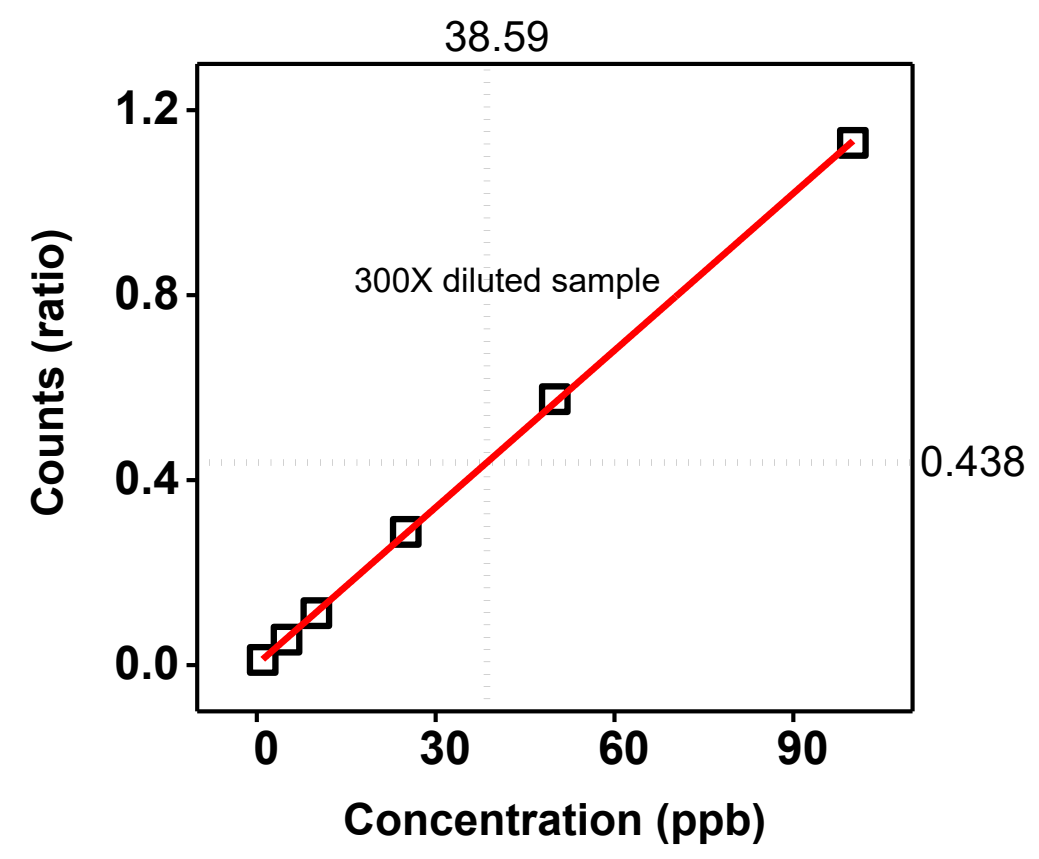

Figure S6. ICP-MS determination of the amount of platinum per spiky PtNC-SWCNT paper electrode prepared via electrodeposition. Calibration curve of standard ${ }^{95} \mathrm{Pt}$ with 300 -fold diluted sample solution. The sample solution was prepared by dissolving the platinum from electrodeposited PtNC-SWCNT paper electrodes using an aqua regia solution.

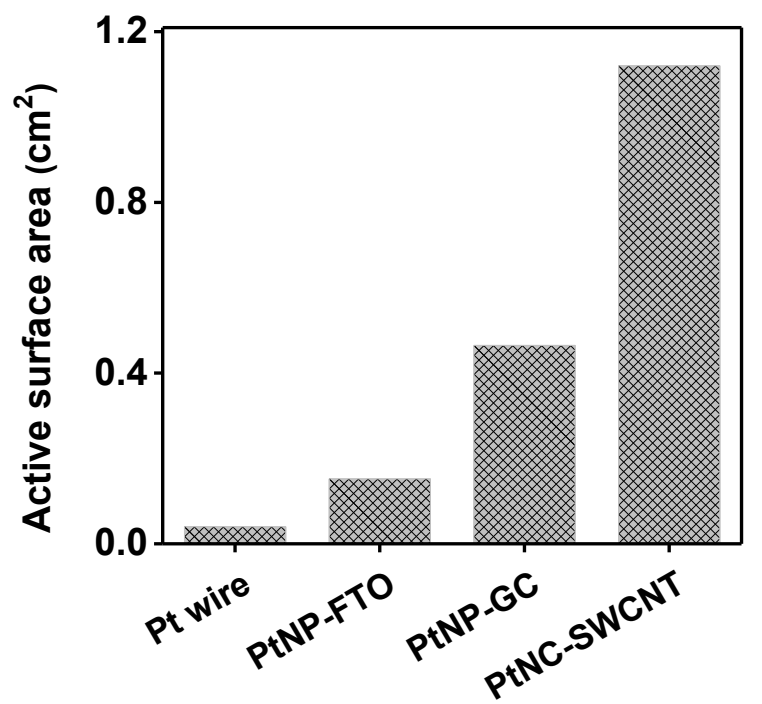

Figure S7. Comparison of electrochemical active surface area of the platinum wire electrode to PtNPmodified fluorine-doped tin oxide electrode (PtNP-FTO), PtNP-modified glassy carbon (PtNP-GC) electrode and PtNC-SWCNT paper electrode. All platinum-coated electrodes were made via electrodeposition. 


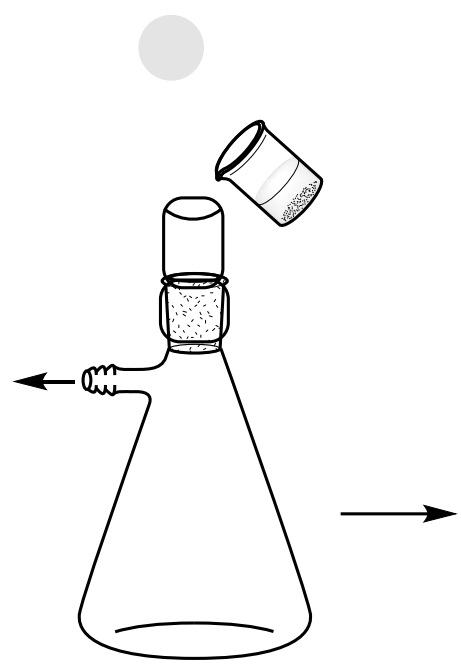

A: SWCNT loading

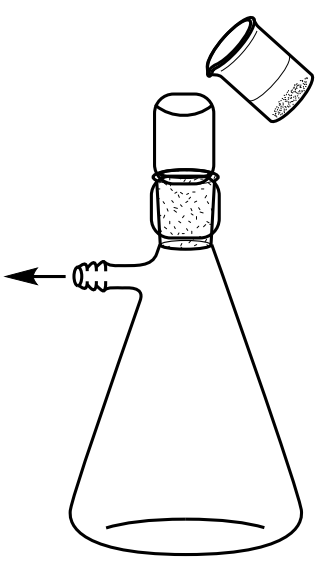

B: Washing

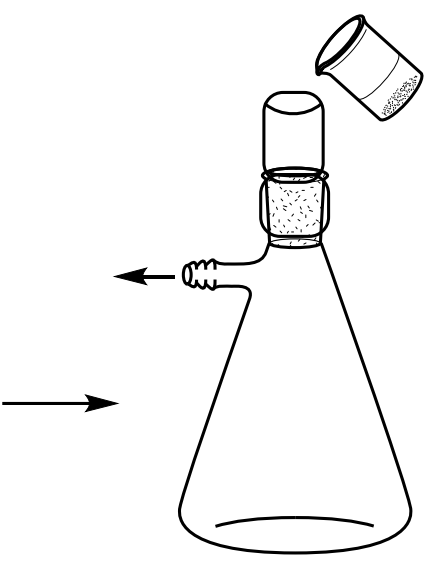

C: PtNP loading

Figure S8. Preparation of PtNP-SWCNT thin film via vacuum filtration. (A) Surfactant-dispersed aqueous solution of SWCNTs was loaded onto a filter membrane to form a porous SWCNT thin film. (B) The surfactant molecule was then washed away using an excess of water. (C) A solution of citrate-capped 29nm PtNPs was finally loaded onto the SWCNT thin film, and a vacuum was applied. The resulting PtNPSWCNT paper film was then used to prepare the electrodes.
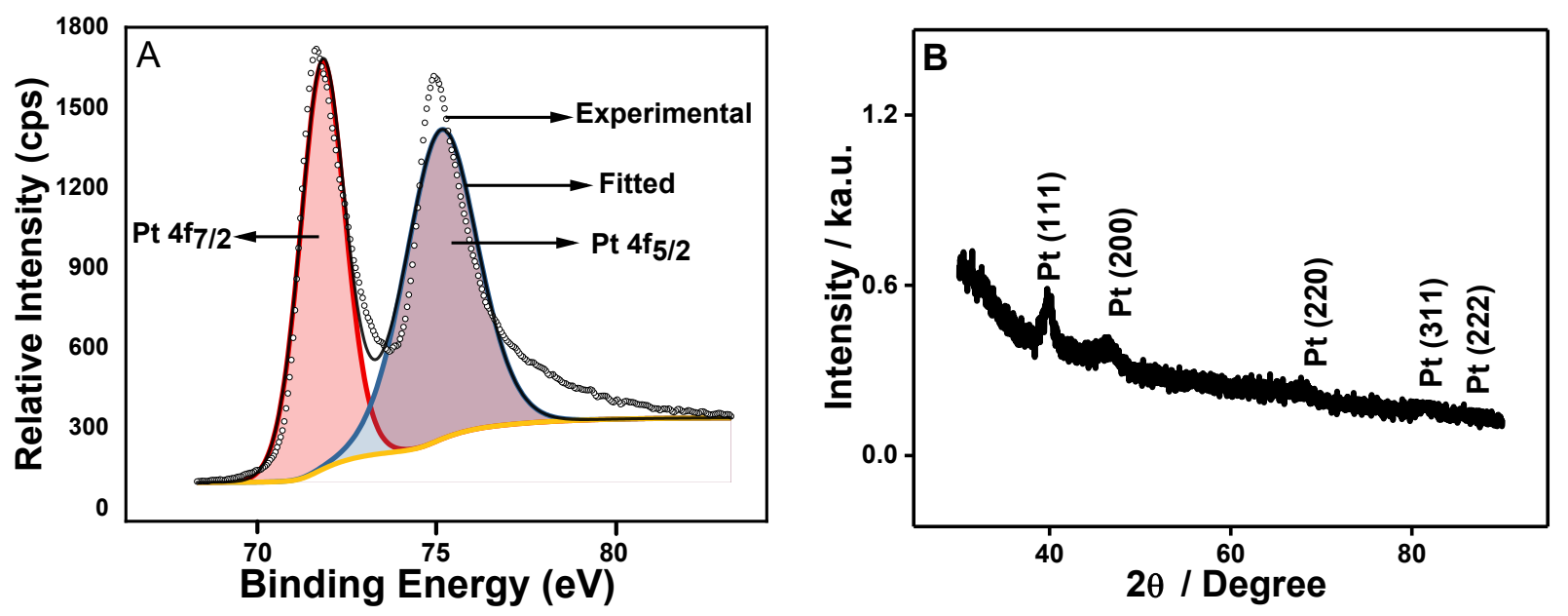

Figure S9. Surface characterization of spherical PtNP-SWCNT paper electrodes prepared by ambient vacuum filtration. (A) XPS spectra showing the oxidation state of platinum and (B) XRD spectra showing the crystalline state of the PtNPs on the surface of the SWCNTs. 


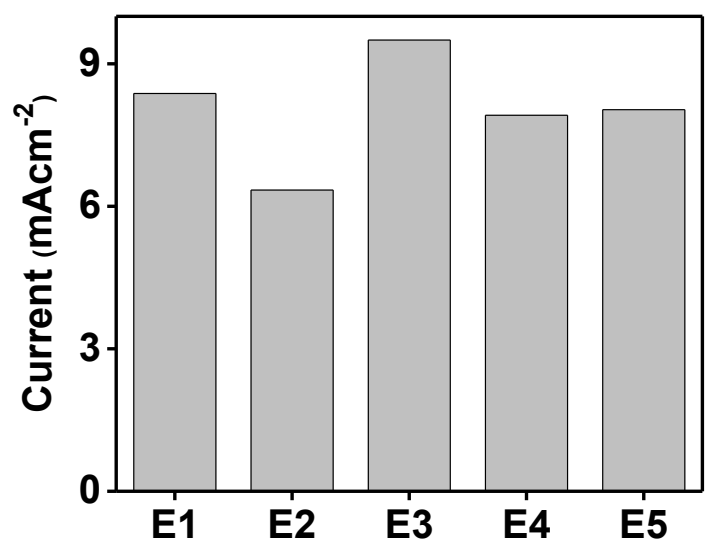

Figure S10. Validation of uniform and reproducible performance from vacuum-filtered PtNP-SWCNT electrodes. We measured the anodic peak current at $0.65 \mathrm{~V}$ of $0.1 \mathrm{M}$ methanol oxidation in $0.1 \mathrm{M} \mathrm{H}_{2} \mathrm{SO}_{4}$ solution for five randomly-chosen electrodes prepared from the same PtNP-SWCNT film. 